\title{
Automatic registration of remote sensing images based on SIFT and fuzzy block matching for change detection
}

\author{
Cai Guo-Rong ${ }^{1,2,3}{ }^{*}$, Li Shao-Zi ${ }^{1,3 *}$, Wu Yun-Dong ${ }^{2}$, Chen Shui-Li ${ }^{2}$, Su Song-Zhi ${ }^{1,3}$ \\ ${ }^{1}$ Department of Cognitive Science, Xiamen University, Xiamen, Fujian, P. R. China 361005 \\ ${ }^{2}$ School of Science, Jimei University, Xiamen, Fujian, P. R. China 361021 \\ ${ }^{3}$ Fujian Key Laboratory of the Brain-like Intelligent Systems (Xiamen University), Xiamen, Fujian, P. R. China,361005
}

Received 11 March 2011

Accepted 23 June 2011

\begin{abstract}
This paper presents an automated image registration approach to detecting changes in multi-temporal remote sensing images. The proposed algorithm is based on the scale invariant feature transform (SIFT) and has two phases. The first phase focuses on SIFT feature extraction and on estimation of image transformation. In the second phase, Structured Local Binary Haar Pattern (SLBHP) combined with a fuzzy similarity measure is then used to build a new and effective block similarity measure for change detection. Experimental results obtained on multi-temporal data sets show that compared with three mainstream block matching algorithms, the proposed algorithm is more effective in dealing with scale, rotation and illumination changes.
\end{abstract}

Keywords: Registration, scale invariant feature transform, fuzzy membership, fuzzy similarity measure, change detection.

\section{Introduction}

As an advanced detection technology, remote sensing has been widely applied in many areas ${ }^{1}$. In particular, change detection is one of the most important applications in the remote sensing society. It is the process of identifying differences in the state of a land cover by analyzing a pair of images acquired on the same geographical area at different times. Since the 1990s, scientists have made remarkable achievements in change detection techniques, including context-sensitive method $^{2}$, GSM approach to automatic change detection in multi-temporal SAR images ${ }^{3}$, ratio analysis ${ }^{4}$, standardized processing $^{5}$, registration noise reduction ${ }^{6}$, MTF-based change detection analysis ${ }^{7}$, automatic unsupervised change detection based on a RGM distribution $^{8}$, etc. Typically, these methods are base on "difference image" and the most widely used operators are subtraction, ratio, and change vector analysis (CVA). Nowadays, change detection techniques have been applied to the investigation of forest resources ${ }^{9}$, land use/land cover dynamic detection ${ }^{10}$, assessment of environment disaster ${ }^{11}$, arrangement of urban growth ${ }^{12}$, and monitoring of national defense ${ }^{13}$, etc.

However, the change detection techniques mentioned above are performed after image registration. Generally a few control points and simple transformation formula are employed in image registration. Obviously, this procedure is simple, but it is time-consuming and not feasible to discern the appropriate control points when a large number of images need to be registered. Therefore, there is a need for automatic registration techniques that require little or no manual intervention.

*Corresponding author: szlg@xmu.edu.cn 
The automatic registration process is usually carried out in four steps ${ }^{14}$, namely, feature detection, feature matching, mapping function design, image transformation and re-sampling. In remote sensing application, there are several works focused on automatic image registration, such as correlation-based automatic registration algorithm ${ }^{15}$, mutual information similarity measure for registration ${ }^{16}$, template matching to search for the corresponding control points between the reference and sensed images ${ }^{17}$. In particular, SURF and hierarchical image matching strategy have been introduced to register remote sensing data which can detect changed area simultaneous with image registration $^{18}{ }^{19}$. It is well known that remote sensing images always acquired through different viewpoints, times and sensors. These factors may result in rotation, illumination changes or even affine transformation in multi-temporal images. In this situation, the efficiency of the methods mentioned above will be greatly reduced. Since the quality of registration is the key factor that dictates the validity and the reliability of the change detection results, effective tools are needed to improve the accuracy of registration in remote-sensing data.

In 1999, scale invariant feature transform (SIFT) ${ }^{20,21}$ was introduced to generate feature points in a full image. These feature points appear invariant to any scaling, rotation or translation of the images. Recently, SIFT descriptor has been introduced to multi-spectral remote image registration and the experimental results demonstrate that SIFT-based automatic registration algorithm can be an effective tool to improve the performance of registration in high resolution remote image $^{22}$. However, due to the diversity of multi-temporal images, it is impossible to design a perfect method to register all pixels in remote sensing images. Namely, even SIFT-based method should take into account registration errors, geometric deformation and noise corruption. In Ref. 23, we proposed a novel feature named Structured Local Binary Haar Pattern (SLBHP), which has been proven to be a good descriptor include the edges, texture and gray information. Therefore, in this paper, SIFT feature descriptor is integrated with SLBHP and fuzzy-based block matching technique to improve image registration. In addition, in order to evaluate the efficiency of the proposed method, the algorithm was used to detect change area in remote sensing images. Experiments carried out on multi-temporal remote sensing images confirm that the improved change detection algorithm is invariance to scale, rotation, and illumination transformations.

This paper is organized as follows: Section 2 provides a brief description of the SIFT feature and feature matching algorithm. Section 3 describes the proposed fuzzy-block matching change detection technique. The dataset used in the experiments and the obtained results are described in Section 4. Finally, in Section 5, conclusions are drawn.

\section{SIFT descriptor and keypoint matching}

\subsection{SIFT local descriptor}

As described in Ref. 20, the construction of SIFT descriptor consists of four major stages, namely, scale-space peak selection, keypoint localization, orientation assignment and keypoint descriptor.

\subsubsection{Detection of scale-space extrema}

The scale space of an image is defined as a function, $L(x, y, \sigma)$, that is produced from the convolution of a variable-scale Gaussian, $G(x, y, \sigma)$, with an input image, $I(x, y)$ :

$$
L(x, y, \sigma)=G(x, y, \sigma) * I(x, y)
$$

where * is the convolution operation, and

$$
G(x, y, \sigma)=\frac{1}{2 \pi \sigma^{2}} e^{-\left(x^{2}+y^{2}\right) / 2 \sigma^{2}} .
$$

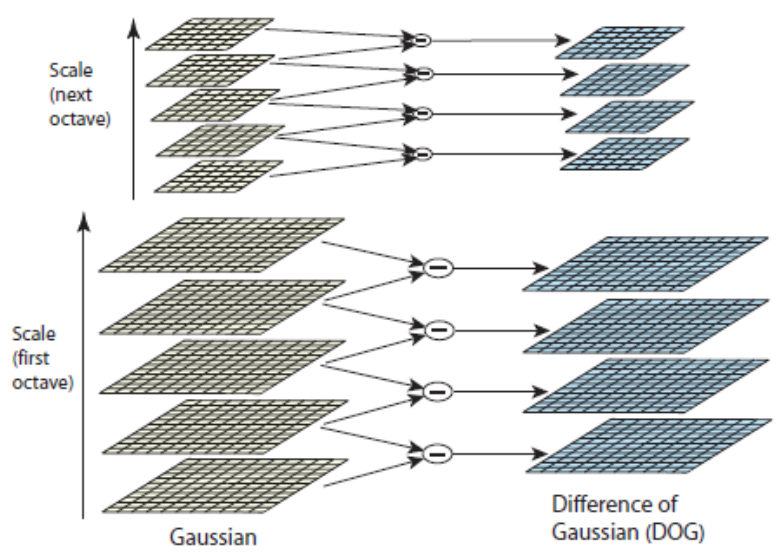

Fig. 1. Difference-of-Gaussian images 
To efficiently detect stable keypoint locations in scale space, SIFT used scale-space extrema in the difference-of-Gaussian function, $D(x, y, \sigma)$, which can be computed from the difference of two nearby scales separated by a constant multiplicative factor $k$, as depicts in Figure 1:

$$
\begin{aligned}
D(x, y, \sigma) & =(G(x, y, k \sigma)-G(x, y, \sigma)) * I(x, y) \\
& =L(x, y, k \sigma)-L(x, y, \sigma)
\end{aligned}
$$

In order to detect the local maxima and minima of $D(x, y, \sigma)$, each sample point is compared to its eight neighbors in the current image and nine neighbors in the scale above and below (see Fig. 2). It is selected as a candidate only if its value is larger than all of these neighbors or smaller than all of them.

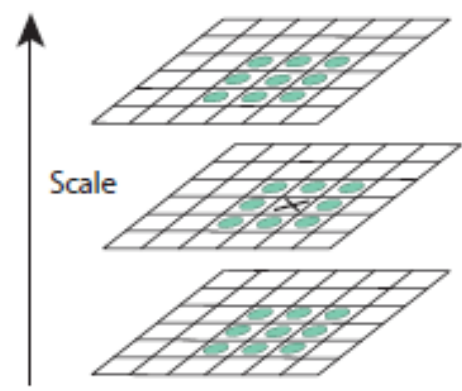

Fig. 2. Extrema of the difference-of-Gaussian images

\subsubsection{Accurate keypoint localization}

Once a keypoint candidate has been found by comparing a pixel to its neighbors, the next step is to perform a detailed fit to the nearby data for location, scale, and ratio of principal curvatures. This information allows points to be rejected points that have low contrast or are poorly localized along an edge.

\subsubsection{Orientation assignment}

For each image sample, $L(x, y)$, the gradient magnitude, $m(x, y)$, and orientation, $\theta(x, y)$, is computed by using pixel differences:

$$
\begin{gathered}
m(x, y)=\sqrt{(L(x+1, y)-L(x-1, y))^{2}+(L(x, y+1)-L(x, y-1))^{2}} \\
\theta(x, y)=\arctan \left(\frac{L(x, y+1)-L(x, y-1)}{L(x+1, y)-L(x-1, y)}\right)
\end{gathered}
$$

An orientation histogram is formed from the gradient orientations of sample points within a region around the keypoint. The orientation histogram has 36 bins covering the 360 degree range of orientations. Each sample added to the histogram is weighted by its gradient magnitude. Peaks in the orientation histogram correspond to dominant directions of local gradients. The highest peak in the histogram is detected, and then any other local peak that is within $80 \%$ of the highest peak is used to also create a keypoint with that orientation.

\subsubsection{Descriptor representation}

Figure 3 illustrates the computation of the keypoint descriptor. First the image gradient magnitudes and orientations are sampled around the keypoint location, using the scale of the keypoint to select the level of Gaussian blur for the image. In order to achieve orientation invariance, the coordinates of the descriptor and the gradient orientations are rotated relative to the keypoint orientation.

The keypoint descriptor is shown on the right side of Figure 3 . The figure shows a $2 \times 2$ array of orientation histograms, whereas it is observed that the best results are achieved with a $4 \times 4$ array of histograms with 8 orientation bins in each. Namely, the feature descriptor vector of each keypoint contains $4 \times 4 \times 8=128$ elements.

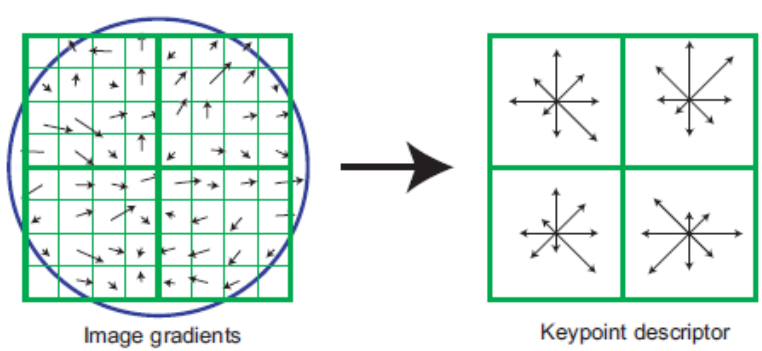

Fig. 3. Keypoint descriptor

\subsection{Keypoint matching}

The best candidate match for each keypoint is found by identifying its nearest neighbor in the keypoints set from reference images. The nearest neighbor is defined as the keypoint with minimum Euclidean distance for the invariant descriptor vector. Since KD-tree algorithm has been proven to be an effective technique for fast searching, and nearest-neighbor queries, KD-tree algorithm is employed for feature matching in this paper. 
Moreover, RANSAC is devised to remove the mismatch keypoint pairs.

Recently, a number of SIFT descriptor variants and extensions, including PCA-SIFT ${ }^{24}$, GLOH (gradient location-orientation histogram) ${ }^{25}$, SURF (speeded up robust features) ${ }^{26}$ and ASIFT (affine SIFT) ${ }^{27}$ have been developed. They claim more robustness and distinctiveness with scaled-down complexity or affine transform. Obviously all the improved SIFT algorithms can be directly apply to registration in remote sensing images.

\section{Change detection in remote sensing images}

This section describes the computation of affine parameters and the re-sampling of affine image. In addition, SLBHP-based fuzzy similarity measure is employed for block matching between reference and affine image to improve image registration. Simultaneously, change area can be determined according to the SLBHP-based similarity score.

\subsection{Affine parameters determination and the construction of affine image}

After the SIFT feature correspondence has been established, the mapping function is to be constructed. One of the most frequently used mapping functions is affine transformation, which can map a parallelogram onto a square. Therefore, we assume that there exists an affine transform between the reference and sensed image. Based on this assumption, the transformation of $\left[\begin{array}{ll}x & y\end{array}\right]^{\mathrm{T}}$ in the reference image to $\left[\begin{array}{ll}u & v\end{array}\right]^{\mathrm{T}}$ in the sensed image can be written as the following equation:

$$
\left[\begin{array}{l}
u \\
v
\end{array}\right]=\left[\begin{array}{ll}
m_{1} & m_{2} \\
m_{3} & m_{4}
\end{array}\right]\left[\begin{array}{l}
x \\
y
\end{array}\right]+\left[\begin{array}{l}
m_{5} \\
m_{6}
\end{array}\right]
$$

where $m_{i} \quad(i=1,2, \ldots, 6)$ are affine parameters.

This equation shows only a single match, in other words, any number of further matches can be added. Specifically, the equation above can be rewritten to gather the unknowns into a column vector:

$$
\left[\begin{array}{cccccc}
x_{1} & y_{1} & 0 & 0 & 1 & 0 \\
0 & 0 & x_{1} & y_{1} & 0 & 1 \\
& & \cdots & & & \\
x_{n} & y_{n} & 0 & 0 & 1 & 0 \\
0 & 0 & x_{n} & y_{n} & 0 & 1
\end{array}\right]\left[\begin{array}{c}
m_{1} \\
m_{2} \\
m_{3} \\
m_{4} \\
m_{5} \\
m_{6}
\end{array}\right]=\left[\begin{array}{c}
u_{1} \\
v_{1} \\
\cdots \\
u_{n} \\
v_{n}
\end{array}\right]
$$

Obviously, at least 3 matches are needed to provide a solution. The linear system can be rewritten as the following equation:

$$
A X=b .
$$

where the parameter vector $X$ can be determined by least-squares solution, as given below:

$$
X=\left(A^{T} A\right)^{-1} A^{T} b .
$$

Therefore, the affine image of the sensed image can be constructed via affine parameters:

- Generate an affine image, $I$, where the size of $I$ is equal to the reference one;

- For each pixel $(u, v)$ in $I$, let $I(u, v)=I^{\prime}$ $\left(m_{1} u+m_{2} v+m_{5}, \quad m_{3} u+m_{4} v+m_{6}\right)$, where $I^{\prime}$ is the sensed image. Since $m_{1} u+m_{2} v+m_{5}$ and $m_{3} u+m_{4} v+m_{6}$ are real numbers, the bilinear interpolation can be employed to determine the gray value of $I^{\prime}\left(m_{1} u+m_{2} v+m_{5}, m_{3} u+m_{4} v+m_{6}\right)$.

\subsection{SLBHP for fuzzy-block matching}

The first step for block matching is the determination of similarity measure. There exist a lot of methods to define similarity function where some include the edges, texture and gray information. In particular, the LBP ${ }^{28}$ operator is one of the best performing texture descriptors and it has proven to be invariance to gray-level changes. In our earlier work, we proposed SLBHP (Structured Local Binary Haar Pattern) ${ }^{23}$ that modified from LBP with Haar wavelet for pixel-based graphics retrieval. In this paper, SLBHP feature was integrated with fuzzy theory to bulid a fuzzy similarity function for block matching between reference and sensed images.

\subsubsection{Structured Local Binary Haar Pattern}

The SLBHP adopts four types of Haar features ${ }^{29}$, which capture the changes of gray values along the horizontal, the vertical and the diagonal directions as shown in Figure 4(a). Only the polarity of Haar feature is involved in SLBHP, while the magnitude is discarded. The polarity relationships are then considered as a binary value as in LBP. It is noted that the number of encoding patterns has been reduced from 256 to 16 . Moreover, SLBHP encoding spatial structure of two adjacent rectangle regions in four-directions. Thus, the SLBHP has compact encoding patterns and incorporates more semantic structure information. 
Let $a_{i}(i=0,1, \cdots, 8)$ be the corresponding gray values for a $3 \times 3$ window at the center pixel $(x, y)$. The SLBHP value of $(x, y)$ is given by the following equation:

$$
S \boldsymbol{B H P}(x, y)=\sum_{p=1}^{4} B\left(H_{p} \otimes N(x, y)\right) \times 2^{p-1}
$$

where

$$
\begin{aligned}
& N(x, y)=\left[\begin{array}{lll}
a_{1} & a_{2} & a_{3} \\
a_{8} & a_{0} & a_{4} \\
a_{7} & a_{6} & a_{5}
\end{array}\right], H_{1}=\left[\begin{array}{rrr}
1 & 1 & 0 \\
1 & 0 & -1 \\
0 & -1 & -1
\end{array}\right], H_{2}=\left[\begin{array}{rrr}
0 & 1 & 1 \\
-1 & 0 & 1 \\
-1 & -1 & 0
\end{array}\right], \\
& H_{3}=\left[\begin{array}{rrr}
1 & 1 & 1 \\
0 & 0 & 0 \\
-1 & -1 & -1
\end{array}\right], H_{4}=\left[\begin{array}{rrr}
-1 & 0 & 1 \\
-1 & 0 & 1 \\
-1 & 0 & 1
\end{array}\right], B(x)= \begin{cases}1 & \text { if }|x|>T \\
0 & \text { otherwise }\end{cases}
\end{aligned}
$$

and $T$ is a threshold (15 in our experiments). By this binary operation, the feature becomes more robust to global lighting changes. It is noted that $H_{p}$ denotes a Haar-like basis function and $H_{p} \otimes N(x, y)$ denotes the difference between the accumulated gray values of the black and red rectangle as shown in Figure 4 (a). Unlike traditional Haar feature, here the rectangles are overlapped with only one pixel as shown in Figure 4(b). An example of SLBHP feature is shown in Figure 4(c).
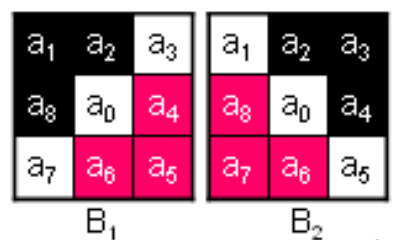

(a)
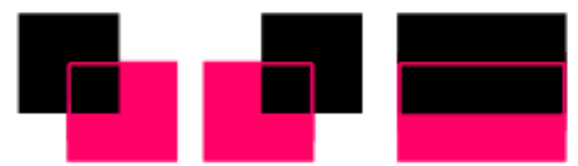

(b)

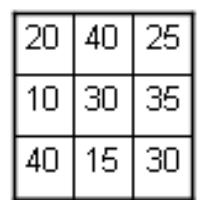

$B_{1}=0,\left|a_{1}+a_{2}+a_{8}-a_{4}-a_{5}-a_{6}\right|=10<T$ $B_{2}=1,\left|a_{2}+a_{3}+a_{4}-a_{6}-a_{7}-a_{8}\right|=35>T$ $\mathrm{B}_{3}=0,\left|\mathrm{a}_{1}+\mathrm{a}_{2}+\mathrm{a}_{3}-\mathrm{a}_{5}-\mathrm{a}_{5}-\mathrm{a}_{7}\right|=0<\mathrm{T}$ $B_{4}=1,\left|a_{1}+a_{7}+a_{8}-a_{3}-a_{4}-a_{5}\right|=20>T$

\section{$\mathrm{SLBHP}=\left(\mathrm{B}_{4} \mathrm{~B}_{3} \mathrm{~B}_{2} \mathrm{~B}_{1}\right)_{2}=(1010)_{2}=10$}

(c)

Fig. 4. An example of SLBHP. (a) Four Haar features; (b) corresponding Haar features with overlapping; (c) an example to compute SLBHP values.

\subsubsection{Fuzzy similarity for block matching}

It is well known that the fuzzy theory has the capability to deal with uncertainty existing in images. For example, fuzzy sets have been successfully introduced to the analysis of landslide susceptibility in remote sensing images ${ }^{30}$, image thresholding ${ }^{31}$ and protein similarity searching ${ }^{32}$. In this paper, SLBHP is integrated with fuzzy theory to construct a fuzzy similarity function for block matching to refine the alignment between images.

The fuzzy membership function of a block $R$ can be defined as the following equation:

$$
f(i)=\frac{\sum_{(x, y) \in R} I\{\operatorname{SLBHP}(x, y)=i\}}{\text { bloksize }}
$$

where $I(P)=\left\{\begin{array}{ll}1 & \text { if } P \text { is true } \\ 0 & \text { if } P \text { is false }\end{array}\right.$ and blocksize denotes the number of pixel in a block $(11 \times 11$ in our experiments). It is worth noting that $f \in[0,1]$ and the fuzzy membership function contains information about the distribution of the local patterns, such as edges and spots, over the image region $R$. Consequently, the similarity measure between blocks is defined as the following equation.

$$
R\left(f_{\text {affine }}, f_{\text {reference }}\right)=\sum_{i} \frac{f_{\text {affine }}(i) \cdot f_{\text {reference }}(i)}{\sqrt{\sum_{j} f_{\text {affine }}(j)^{2} \cdot \sum_{j} f_{\text {reference }}(j)^{2}}}
$$

where $f_{\text {affine }}$ and $f_{\text {reference }}$ are the fuzzy membership function of blocks in the affine and reference image, respectively. $i, j \in[0,15]$ refer to the value of SLBHP.

Finally, the $11 \times 11$ neighbors of each pixel are generated as a block in the reference image. The block is devised to search in the corresponding pixel's $30 \times 30$ neighborhood window in the affine image. As a result, each pixel will attach fuzzy similarity scores in the sub-window. If the highest score is lower than a specified threshold, the pixel will be denoted as a changed point.

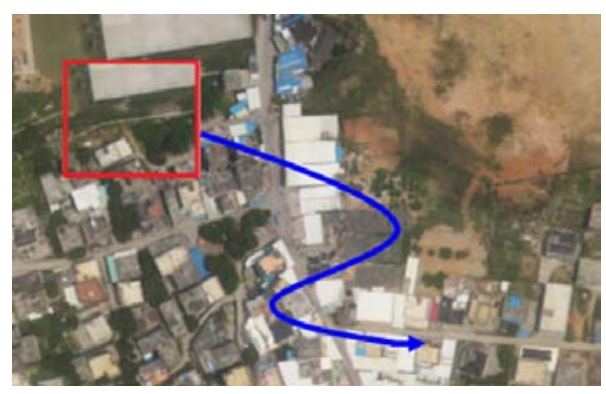

Fig. 5. Block matching in sub-window 


\subsection{Algorithm summary}

Step1: Generate SIFT features of the reference and sensed image;

Step2: KD-tree algorithm for SIFT feature matching;

Step3: RANSAC to remove mismatch points;

Step4: Compute affine parameters via least-squares solution;

Step5: Construct an affine image of the sensed image according to the affine transform matrix;
Step6: Generate membership function for each pixel in the reference image based on SLBHP.

Step7: Match in the corresponding sub-window in the affine image and denote changed area in terms of the fuzzy similarity measure.

Figure 6 depicts the flowchart of the proposed algorithm.

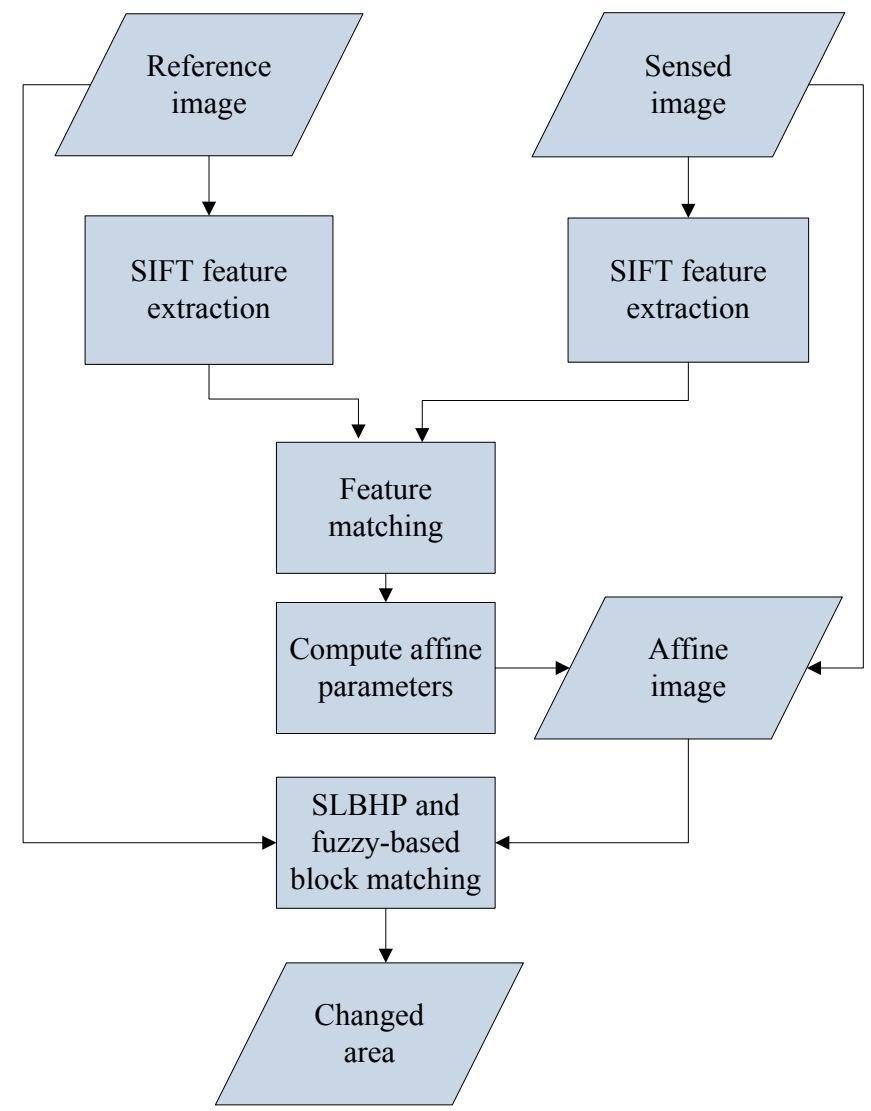

Fig. 6. The proposed SIFT and SLBHP-based block matching change detection algorithm

\section{Experimental results}

\subsection{Description of the experiments}

In this section, three tests have been conducted using several remote sensing images to illustrate the feasibility and the robustness of the proposed technique. The simulated dataset was acquired by unman low altitude remote sensing system of our lab. In particular, a section of the scene acquired in an urban area near
Xiamen city of Fujian Province, P. R. China, was selected.

Fig. 7(a) and (b) show the reference and the sensed image acquired in 2009 and 2010, respectively. As is readily apparent (see the middle part of the sensed image), a new building occupied a notable portion given the two dates considered. The available ground truth map (see Fig. 7(c)) is used to assess change-detection errors. 


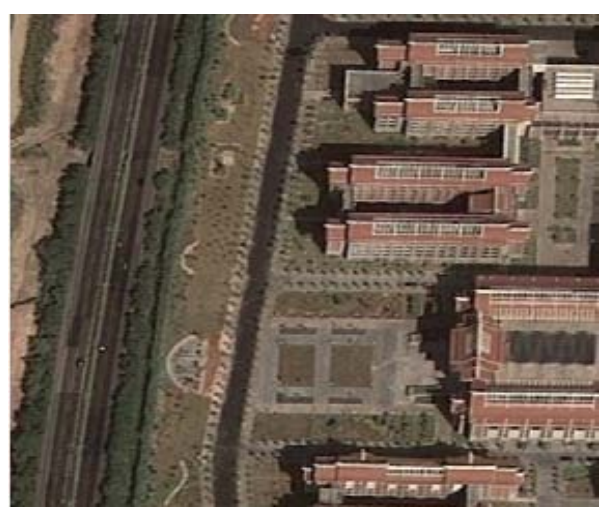

(a)

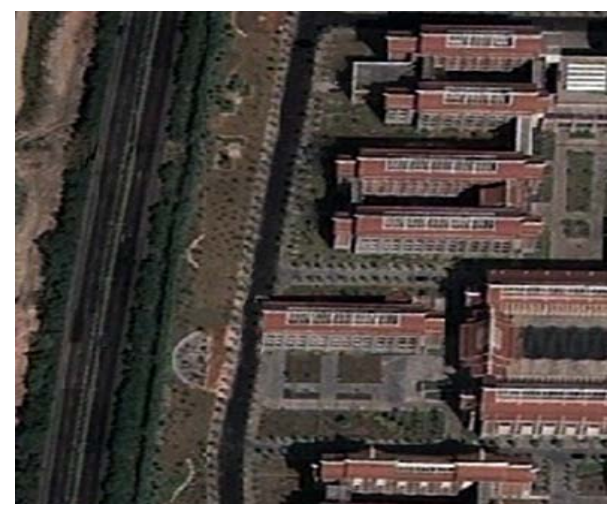

(b)

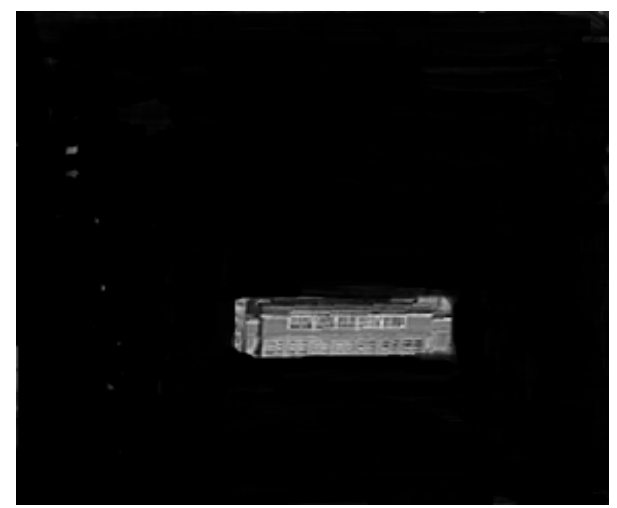

(c)

Fig. 7. (a) Reference image, (b) Sensed image, (c) Ground-truth map of the changed area.
In the first experiment, the sensed image is rotated to evaluate the performance of rotation invariance of the SLBHP-based block matching strategy. In the second experiment, $2 \times 2$ sub-sampling of sensed image is used to present the invariance in scale change of our approach.

The third experiment focuses on the invariance of gray-level changes. The results obtained by the proposed technique will be compared with other three block matching strategies, namely, square difference, correlation and correlation coefficient, as given below:

- Square difference

$$
R_{1}(x, y)=\frac{\sum_{x^{\prime}, y^{\prime}}\left[T\left(x^{\prime}, y^{\prime}\right)-I\left(x+x^{\prime}, y+y^{\prime}\right)\right]^{2}}{\sqrt{\sum_{x^{\prime}, y^{\prime}} T\left(x^{\prime}, y^{\prime}\right)^{2} \sum_{x^{\prime}, y^{\prime}} I\left(x+x^{\prime}, y+y^{\prime}\right)^{2}}}
$$

- Correlation

$$
R_{2}(x, y)=\frac{\sum_{x^{\prime}, y^{\prime}}\left[T\left(x^{\prime}, y^{\prime}\right) \cdot I\left(x+x^{\prime}, y+y^{\prime}\right)\right]}{\sqrt{\sum_{x^{\prime}, y^{\prime}} T\left(x^{\prime}, y^{\prime}\right)^{2} \sum_{x^{\prime}, y^{\prime}} I\left(x+x^{\prime}, y+y^{\prime}\right)^{2}}}
$$

- Correlation coefficient

$$
R_{3}(x, y)=\frac{\sum_{x^{\prime}, y^{\prime}}\left[T^{\prime}\left(x^{\prime}, y^{\prime}\right) \cdot I^{\prime}\left(x+x^{\prime}, y+y^{\prime}\right)\right]}{\sqrt{\sum_{x^{\prime}, y^{\prime}} T^{\prime}\left(x^{\prime}, y^{\prime}\right)^{2} \sum_{x^{\prime}, y^{\prime}} I^{\prime}\left(x+x^{\prime}, y+y^{\prime}\right)^{2}}}
$$

where $T^{\prime}\left(x^{\prime}, y^{\prime}\right)=T\left(x^{\prime}, y^{\prime}\right)-\frac{1}{w \cdot h} \sum_{x^{\prime \prime}, y^{\prime \prime}} T\left(x^{\prime \prime}, y^{\prime \prime}\right)$,

$I^{\prime}\left(x+x^{\prime}, y+y^{\prime}\right)=I\left(x+x^{\prime}, y+y^{\prime}\right)-\frac{1}{w \cdot h} \sum_{x^{\prime \prime}, y^{\prime \prime}} I\left(x+x^{\prime \prime}, y+y^{\prime \prime}\right)$

are mean template brightness and mean patch brightness, respectively.

\subsection{Rotation and scale invariant}

The first experiment is related to the problem of the analysis of rotation invariant of the proposed approach. Therefore, the sensed image was being rotated 90 degrees and 45 degrees clockwise (see Fig. 8(a) and Fig. 8(b)). The change results are show in Fig. 8(c) and Fig. 8(d), respectively. It is worth noting that the proposed technique provided quite accurate estimates of the change area Namely, the SIFT-SLBHP method is invariance to rotation. 


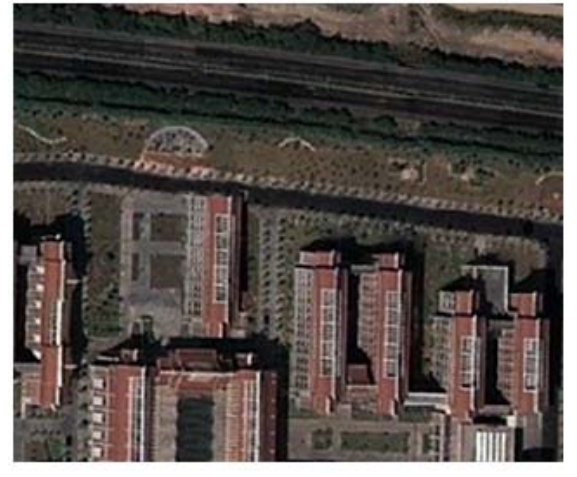

(a)

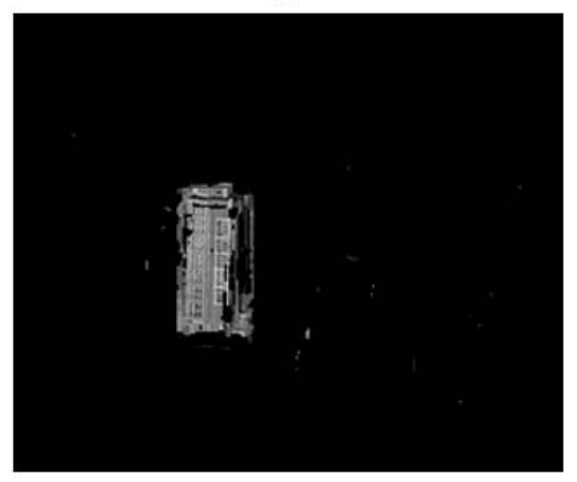

(c)

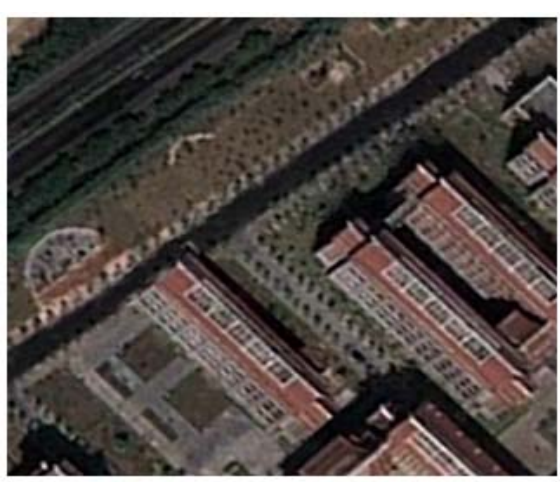

(b)

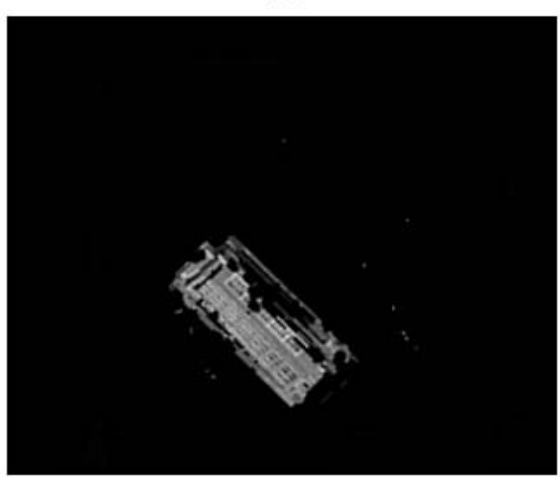

(d)

Fig. 8. (a) Reference image, (b) Sensed image, (c) Ground-truth map of the changed area. (d) Reference map of the changed area.

The second experiment conducted on a $2 \times 2$ sub-sampling of the sensed image (Fig. 9(a)). Our goal here is to demonstrate the scale invariant of SIFT approach. Figure 9(b) illustrates the obtained change detection map in this simulation. The result is a little worse than we achieved in the first experiment. The main reason is that some information of the sensed image is lost during the construction of the affine map. Nonetheless, as shown in Figure 9(b), the result detect by our approach still encouraging.

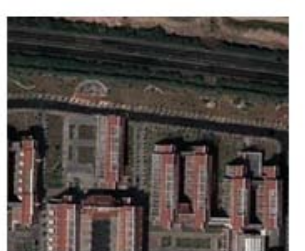

(a)

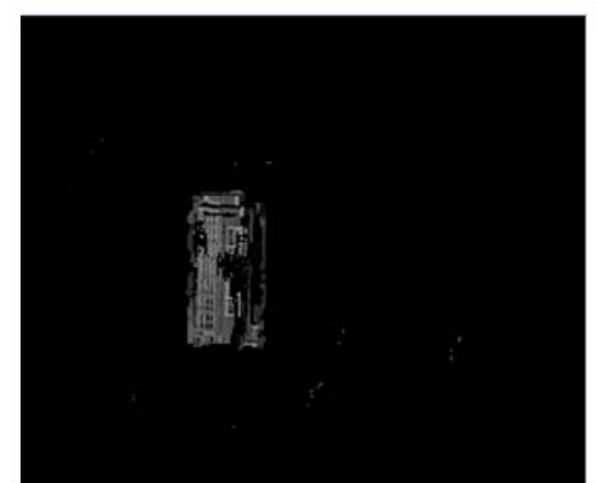

(b)

Fig. 9. (a) $2 \times 2$ sub-sampling of the sensed image, (b) Reference map of the changed area. 


\section{3. gray-level invariant}

Firstly, the sensed image was converted by reducing gray value of each pixel to $1 / 3$ of its origin one (see Figure 10(a)). The change detection maps obtained by the square difference, correlation, correlation coefficient and our SLBHP similarity measure are given in Figure 10(c) to10(e), respectively.

From figure $10(\mathrm{c})$ to $10(\mathrm{e})$, one can see clearly that there were many isolated pixels or some small isolated areas produced by the former three methods, whereas our approach obtained quite ideal result. In particular, as shown in Figure 10(b), if we reduce the gray value of each pixel by half, the former three methods all fail (Figure 10(g)-10(i)). On the contrary, the proposed SLBHP block matching can detect change place more encouraging, as shown in Figure 10(j). These results verify that the proposed method is effective in gray-level changes.

\section{Conclusions and discussion}

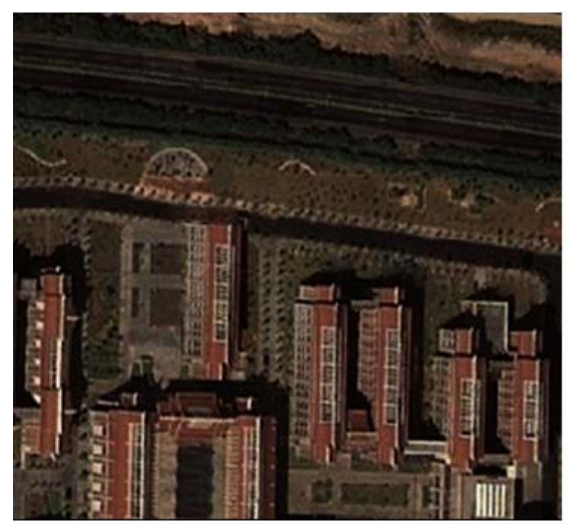

(a)

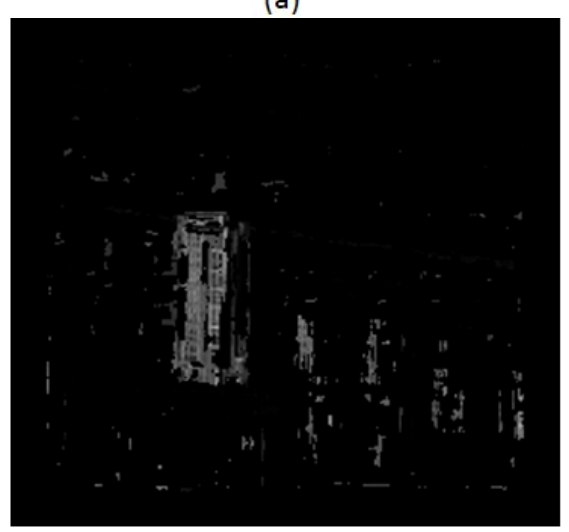

(c)
A novel automatic remote-sensing image registration and change detection approach based on SIFT and SLBHP has been proposed in this paper. The technique employed SIFT feature descriptor to improve image registration and SLBHP-based block matching for change detection. The experimental results confirmed the effectiveness of the proposed approach. In particular, the proposed technique is invariance to scale, rotation, and gray-level transformations.

As a final remark, it is important to point out that, although the proposed method can be used to design a system for the change detection in multi-temporal remote-sensing images, potentially, it is general and can be considered for any pair of large-size multi-temporal images. For this reason, as future developments of this work, we plan to extend the experimental investigation to other data sets and change-detection problems. In addition, our algorithm will be compared with other state-of-the-art methodologies of registration to better assess the robustness of the approach in different scenarios.

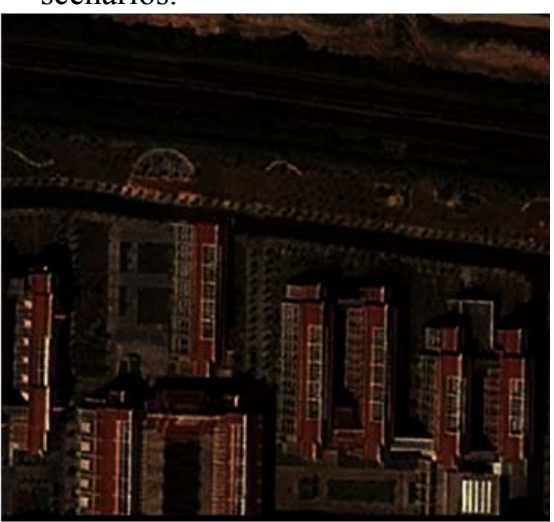

(b)

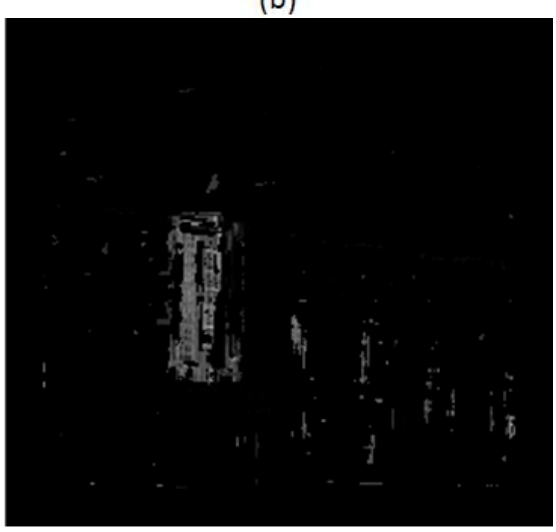

(d) 


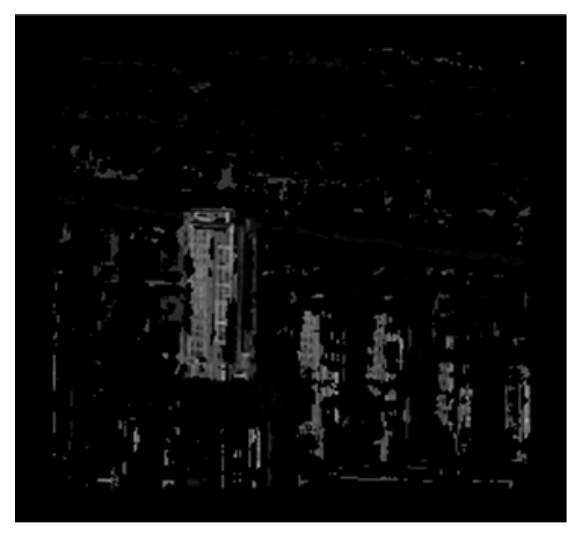

(e)

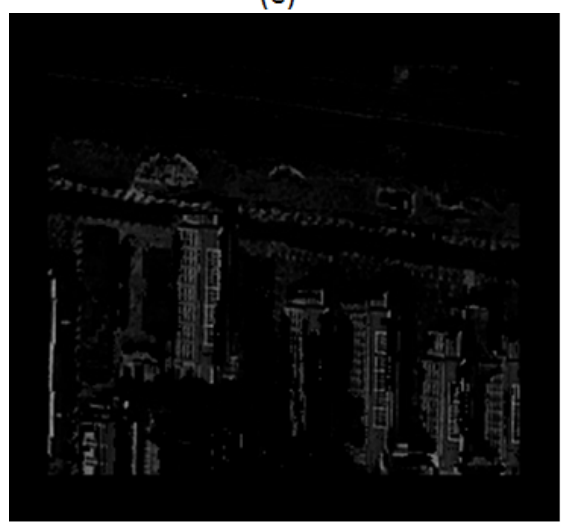

(g)

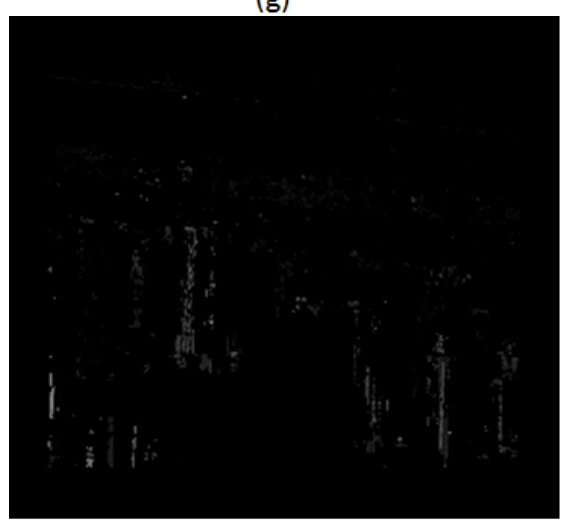

(i)

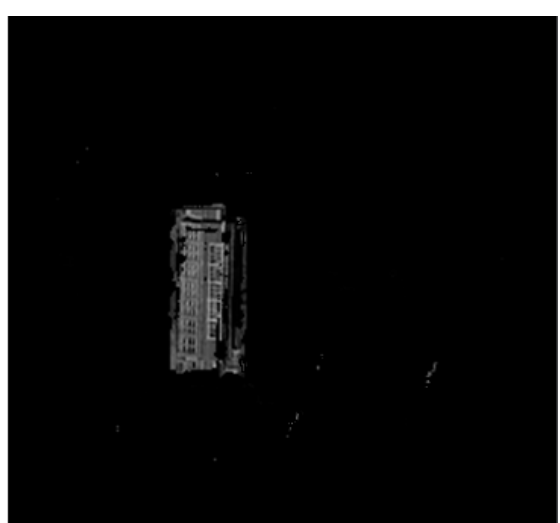

(f)

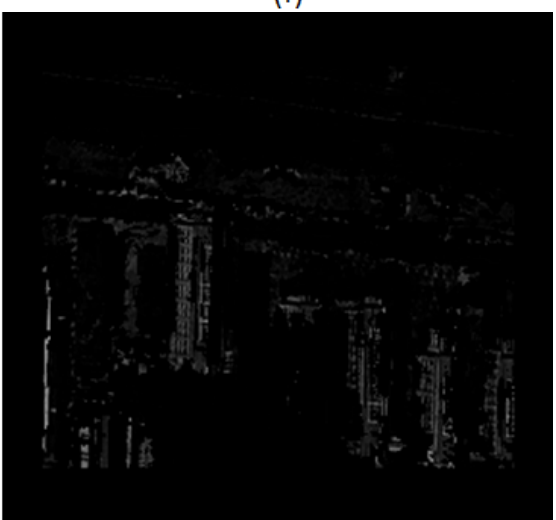

(h)

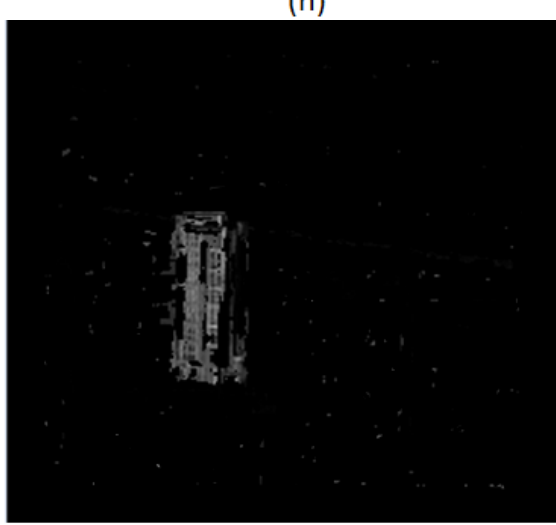

(j)

Fig. 10. (a) two third gray value of the sensed image, (b) half gray value of the sensed image, (c), (d), (e), (f) Reference maps of the changed area of Fig. 10 (a) acquired by square difference, correlation, correlation coefficient and the proposed method, respectively. (g), (h), (i), (j) Reference maps of the changed area of Fig. 10 (b) acquired by square difference, correlation, correlation coefficient and the proposed method, respectively.

\section{Acknowledgements}

This work was supported by National Nature Science Foundation of China (No.60873179); Doctoral Program Foundation of Institutions of Higher Education of China (No. 20090121110032); Shenzhen Science and
Technology Research Foundation (No.JC200903180630A, ZYB200907110169A); The Natural Science Foundation of Xiamen City, PRC (3502Z2093018). The Projects of Education Department of Fujian Province (JK2009017, JK2010031, JA10196). 


\section{References}

1. Z. Wang, S. Li, Y. Lv and K. Yang, Remote sensing image enhancement based on orthogonal wavelet transformation analysis and pseudo-color processing, International Journal of Computational Intelligence Systems. 3(6) (2010) 745-753.

2. S. Ghosh, L. Bruzzone, S. Patra, F. Bovolo and A. Ghosh, A context-sensitive technique for unsupervised change detection based on Hopfield-type neural networks, IEEE Transaction on Geoscience and Remote Sensing. 45(3) (2007) 778-789.

3. Y. Bazi, L. Bruzzone and F. Melgani, An Unsupervised Approach Based on the Generalized Gaussian Model to Automatic Change Detection in Multi-temporal SAR Images, IEEE Transaction on Geoscience and Remote Sensing. 43(4) (2005) 874-886.

4. E. Rignot and Z. Jvan, Change detection techniques for ERS-1 SAR data, IEEE Transaction on Geoscience and Remote Sensing. 31(4) (1993) 896-906.

5. P. Coppin and M. Bauer, Processing of multi-temporal Landsat TM imagery to optimize extraction of forest cover change features, IEEE Transaction on Geoscience and Remote Sensing. 32(4) (1994) 918-927.

6. L. Bruzzone and R. Cossu, An adaptive approach to reducing registration noise effects in unsupervised change detection, IEEE Transaction on Geoscience and Remote Sensing.41(11) (2003) 2455-2465.

7. G. R. Ma, P. X. Li and Q. Q. Qin, Based on Fusion and GGM Change Detection Approach of remote Sensing Images, Journal of Remote Sensing. 10(6) (2006) 847-853.

8. G. Wang, Y. Wang and L. Jiao, Adaptive spatial neighborhood analysis and Rayleigh-Gauss distribution fitting for change detection in multi-temporal remote sensing images, Journal of Remote Sensing.13(4) (2009) 631-638.

9. J. E. S. Fransson, F. Walter and K. Blennow, Gustavsson $A$ and LMH Ulander, Detection of storm-damaged forested areas using Airborne CARABAS-II VHF SAR image data, IEEE Transaction on Geoscience and Remote Sensing. 40(10) (2002) 2170-2175.

10. G. M. Foody, Monitoring the magnitude of land-cover change around the southern limits of the Sahara, Photogrammetric Engineering and Remote Sensing. 67(7) (2001) 841-847.

11. F. Bovolo and L. Bruzzone, A split-based approach to unsupervised change detection in large-size multi-temporal images: application to Tsunami-damage assessment, IEEE Transaction on Geoscience and Remote Sensing. 45(6) (2007) 658-1670.

12. Y. Li, S. Zhao, K. Zhao, P. Xie and J. Fang and Land-cover changes in an Urban Lake Watershed in a Mega-city, Central China, Environ. Monit. Assess. 115(1-3) (2006) 349-359.

13. D. Potin, P. Vanheeghe, E. Duflos and M. Davy, An abrupt change detection algorithm for buried landmines localization, IEEE Transaction on Geoscience and Remote Sensing. 44(2) (2006) 260-272.
14. B. Zitova' and J. Flusser, Image registration methods: a survey, Image and Vision Computing. 21 (2003) 977-1000.

15. J. Le Moigne, An automated parallel image registration technique based on the correlation of wavelet features, IEEE Transaction on Geoscience and Remote Sensing. 40(8) (2002) 1849-1864.

16. H. Chen, P. K. Varshney and M. K. Arora, Performance of Mutual Information Similarity Measure for Registration of Multi-temporal Remote Sensing Images, IEEE Transaction on Geoscience and Remote Sensing. 40(11) (2003) 2445-2454.

17. Y. Bentoutou, N. Taleb, K. Kpalma, and J. Ronsin, An automatic image registration for applications in remote sensing, IEEE Transaction on Geoscience and Remote Sensing. 43(9) (2005) 2127-2137.

18. A. Brook and Eyal Ben-Dor, Automatic Registration of Airborne and Spaceborne Images by Topology Map Matching with SURF Processor Algorithm, Remote Sensing. 3(1) (2011) 65-82.

19. C. Zhang and C. S. Fraser, Automated registration of high-resolution satellite imagery for change detection, Int. Archives of Photogrammetry, Remote Sensing and Spatial Information Sciences. 36(1) (2005) 3-8.

20. D.G. Lowe, Object recognition from local scale-invariant features, in Proc. of the Int. Conf. Computer Vision, (Corfu, Greece, 1999), pp. 1150 1157.

21. D.G. Lowe, Distinctive image features from scale-invariant key points, International Journal of Computer Vision. 60(2) (2004) 91-110.

22. Z. Yi, C. Zhiguo and X. Yang, Multi-spectral remote image registration based on SIFT, Electronics Letters. 44(2) (2008) 107-108.

23. S. Su, S. Chen, S. Li and D. Duh, Structured local binary Haar pattern for pixel-based graphics retrieval, Electronics Letters. 46(14) (2010) 996-998.

24. Y. Ke and R. Sukthankar, PCA-SIFT: A more distinctive representation for local image descriptors. in Proc. of the IEEE Conf. on Computer Vision and Pattern Recognition, 2004, 2:506-513.

25. K. Mikolajczyk and C. Schmid, A performance evaluation of local descriptors, IEEE Trans. PAMI. 27(10) (2005) 1615-1630.

26. H. Bay, T. Tuytelaars, and L. Van Gool, Surf: Speeded up robust features, in Proc. of the European Conference on Computer Vision, 2006, 1:404-417.

27. J. M. Morel and G.Yu, ASIFT: A new framework for fully affine invariant image comparison, SIAM Journal on Imaging Sciences. 2(2) (2009) 438-469.

28. T. Ojala, M. Pietiknen and D. Harwood, A comparative study of texture measures with classification based on feature distributions, Pattern Recognition. 29 (1) (1996) 51-59.

29. P. Viola and M. Jones, Robust real-time face detection, International Journal of Computer Vision. 57(2) (2004) 137-154.

30. B. Pradhan, Application of an advanced fuzzy logic model for landslide susceptibility analysis, International 
Journal of Computational Intelligence Systems. 3(3) (2010) 370-381.

31. T. Wu and K. Qin, Comparative study of smage thresholding using Type-2 fuzzy sets and cloud model, International Journal of Computational Intelligence Systems. 3(1) (2010) 61-73.

32. D. Mrozek and B. Małysiak-Mrozek An improved method for protein similarity searching by alignment of fuzzy energy signatures, International Journal of Computational Intelligence Systems. 4(1) (2011) 75-88. 